\section{Serum monoclonal component in chronic lymphocytic leukemia: baseline correlations and prognostic impact}

The first report analyzing the presence of a serum monoclonal component (sMC) in patients with lymphoid malignancies was published in 1957. ${ }^{1}$ Since then, the identification of a sMC in other lymphoid neoplasms has awakened interest, mainly due to its relationship with Bcell biology and its adverse prognostic impact in some entities, such as diffuse large B-cell lymphoma. ${ }^{2-4}$ To date, five reports have employed serum immunofixation electrophoresis (sIFE) to study the presence of a sMC in chronic lymphocytic leukemia (CLL) ${ }^{5-10}$ In the largest of these studies, containing 133 patients, $\mathrm{Xu}$ and colleagues reported a prevalence of $20 \%$, and correlated the presence of a sMC with advanced stage, adverse prognostic features, and worse overall survival (OS). ${ }^{9}$ To our knowledge, the present study represents the largest series analyzing the prevalence and prognostic impact of a sMC in CLL. Found in $30 \%$ of patients at diagnosis, it is associated with clinical, biological and genetic adverse prognostic features, and shorter OS and relative survival, with a comparable time to first treatment (TTFT), risk of Richter syndrome (RRS), and risk of second malignancies (RSM).

We studied 548 patients diagnosed with CLL ( $n=340)$, CLL-type monoclonal B-cell lymphocytosis (MBL, $\mathrm{n}=108$ ), or small lymphocytic lymphoma (SLL, $\mathrm{n}=93$ ) at a single institution between 1997 and 2018, with available data on sIFE at diagnosis. The study was conducted according to the Hospital Clínic de Barcelona Institutional Review Board and in accordance with the Declaration of Helsinki. Serum protein electrophoresis
(sPEP) and sIFE were performed at diagnosis in all patients. A non-measurable sMC was defined as a positive sIFE but normal sPEP. A biclonal sIFE was defined as the presence of two heavy or light chains on the sIFE. Immunoparesis was defined as a decreased level of at least one of the three immunoglobulin (Ig) classes. Light chains were considered concordant if the light chains of the sIFE matched the light chain restriction by flow cytometry of the peripheral blood and/or bone marrow.

In order to estimate TTFT, RRS and RSM, in which death without the primary event is possible, cumulative incidence was calculated (cmprsk package, $\mathrm{R}$ software, Vienna, Austria) and compared by use of Gray's test. In order to compare the OS observed in our cohort with that of the general population, patients were matched by age and sex with Spanish individuals from the Human Mortality Database,$^{11}$ which provides an estimate of the cause-specific survival through relative survival analysis (relsurv package, R software, Vienna, Austria). Excess mortality (also called survival reduction), expressed as a percentage, was calculated with the following formula: [1-(cohort survival/population survival)] x 100, and was intended to reflect the reduction in life expectancy with respect to the general population. Variables that had a significant impact on OS were used to construct a multivariate Cox proportional hazards regression model. $P$-values $<0.05$ were considered to indicate statistical significance.

Baseline characteristics of the patients are shown in Table 1. One hundred and sixty-five patients $(30 \%)$ had $\mathrm{a}+\mathrm{sIFE}$ at diagnosis. Patients with a +sIFE were significantly older (median age 70 years [y] vs. $66 \mathrm{y}, P=0.007$ ), and the prevalence of a +sIFE increased with age. Patients diagnosed with SLL had a higher prevalence of a + sIFE

Table 1. Baseline characteristics of the patients

\begin{tabular}{|c|c|c|c|c|}
\hline \multirow[t]{2}{*}{ Characteristic } & \multirow[b]{2}{*}{$\begin{array}{c}\text { All patients } \\
(n=548)\end{array}$} & \multicolumn{3}{|c|}{ Serum immunofixation } \\
\hline & & $\begin{array}{c}\text { Negative } \\
(\mathrm{n}=383,70 \%)\end{array}$ & $\begin{array}{c}\text { Positive } \\
(n=165,30 \%)\end{array}$ & $P$ \\
\hline Age in years, median (range) & $67(30-97)$ & $66(30-92)$ & $70(32-97)$ & 0.007 \\
\hline Male sex, n (\%) & $324(59)$ & $223(58)$ & $101(61)$ & NS \\
\hline ECOG PS $\geq 1$, n (\%) & $44(9)$ & $19(5)$ & $25(16)$ & $<0.001$ \\
\hline Lymphadenopathy (CT), n (\%) & $191(57)$ & $125(52)$ & $66(70)$ & 0.003 \\
\hline B symptoms, n (\%) & $20(4)$ & $13(4)$ & $7(5)$ & NS \\
\hline Binet stage C, n (\%) & $26(5)$ & $12(3)$ & $14(9)$ & 0.007 \\
\hline Rai stage III-IV, n (\%) & $30(6)$ & $12(3)$ & $18(11)$ & $<0.001$ \\
\hline $\beta 2$-microglobulin above ULN, n (\%) & $257(48)$ & $158(42)$ & $99(62)$ & $<0.001$ \\
\hline $\begin{array}{l}\text { FISH [n=493 (90\%)] } \\
\text { normal, n (\%) } \\
\text { del(13q), n (\%) } \\
+12, \mathrm{n}(\%) \\
\text { del(11q), n (\%) } \\
\text { del(17p), n (\%) }\end{array}$ & $\begin{array}{l}145(29) \\
203(41) \\
75(15) \\
49(10) \\
21(4)\end{array}$ & $\begin{array}{c}105(31) \\
146(43) \\
51(15) \\
229(9) \\
9(3)\end{array}$ & $\begin{array}{l}40(26) \\
57(37) \\
24(16) \\
20(13) \\
12(8)\end{array}$ & 0.032 \\
\hline Unmutated IGHV genes, n (\%) [n=333 (61\%)] & $139(42)$ & $87(37)$ & $52(53)$ & 0.009 \\
\hline Abnormal ATM gene, $\mathrm{n}(\%)$ [n=237 (43\%)] & $17(7)$ & $11(6)$ & $6(9)$ & NS \\
\hline Mutated NOTCHI gene, $\mathrm{n}(\%)$ [n=288 (53\%)] & $31(11)$ & $19(9)$ & $12(15)$ & NS \\
\hline Mutated SF3B1 gene, $\mathrm{n}(\%)$ [n=276 (50\%)] & $26(9)$ & $17(9)$ & $9(12)$ & NS \\
\hline Abnormal TP53 gene, $n(\%)[n=268$ (49\%)] & $20(8)$ & $14(7)$ & $6(8)$ & NS \\
\hline Abnormal BIRC3 gene, $\mathrm{n}(\%)$ [n=102 (19\%)] & $1(1)$ & $1(1)$ & 0 & NS \\
\hline Mutated MYD88 gene, $\mathrm{n}(\%)[\mathrm{n}=102(19 \%)]$ & $4(4)$ & $3(4)$ & $1(3)$ & NS \\
\hline
\end{tabular}


( $45 \%$ vs. $27 \%$ and $25 \%$ for CLL and MBL, respectively). The presence of a + sIFE was associated with a worse Eastern Cooperative Oncology Group performance status (ECOG PS), higher frequency of lymphadenopathy, more advanced Binet and Rai stages, and higher $\beta_{2}$-microglobulin (B2M) levels (62 vs. $42 \%, P<0.001)$. The distribution of fluorescence in situ hybridization (FISH) abnormalities was significantly different according to the sIFE, with a higher frequency of favorable-risk FISH in -sIFE patients, and of high-risk abnormalities in +sIFE patients $(P=0.032)$. Likewise, the proportion of patients with unmutated immunoglobulin heavy chain gene (IGHV) genes was significantly higher among patients with a + sIFE (53 vs. $37 \%, P=0.009$ ). No significant differences were seen in the mutation/deletion rate of $A T M$, NOTCH1, SF3B1, TP53, MYD88, or BIRC3 according to the sIFE.
The proportion of patients with immunoparesis did not differ according to the sIFE. Among the 165 patients with a +sIFE at diagnosis, IgM- $\kappa$ was the most common isotype $(25 \%)$, followed by IgG- $\kappa(22 \%)$, and IgG- $\lambda$ (21\%). The frequency of other isotypes was: $\operatorname{Ig} M-\lambda(8 \%)$, $\operatorname{IgA}-\kappa(2 \%), \operatorname{IgA}-\lambda(2 \%), \kappa$ free-light chains ( - -FLC, $1 \%)$, $\lambda$-FLC $(6 \%)$, and bi/triclonal $(11 \%)$. Among the cases in which the quantification of the sMC was available for review, most had a non-measurable sMC (positive sIFE but normal sPEP). In the remaining eight cases, the median sMC was $4.3 \mathrm{~g} / \mathrm{L}$ (range, 2-9.8). The information between the light chain of the serum Ig and the light chain restriction by flow cytometry was compared in 132 cases with a +sIFE: $72 \%$ were concordant and $28 \%$ were discordant. When concordance was further analyzed considering the sIFE isotype, it was found to be higher for IgM cases (89\%). Protein and immunochemical features

Table 2. Treatment, response, and outcomes.

\begin{tabular}{|c|c|c|c|c|}
\hline \multirow[t]{2}{*}{ Characteristic } & \multirow{2}{*}{$\begin{array}{c}\text { All } \\
\text { patients }\end{array}$} & \multicolumn{3}{|c|}{ Serum immunofixation } \\
\hline & & Negative & Positive & $\mathbf{P}$ \\
\hline 10-y probability of requiring treatment, & 49 & 48 & 52 & NS \\
\hline$\%(95 \% \mathrm{CI})$ & $(44-54)$ & $(42-53)$ & $(43-60)$ & \\
\hline \multicolumn{4}{|l|}{ Frontline treatment [ $\mathrm{n}=230(42 \%)], \mathrm{n}(\%)$} & \multirow[t]{6}{*}{ NS } \\
\hline Alkylating agents $+/$ - rituximab & $62(27)$ & $44(29)$ & $18(24)$ & \\
\hline Purine analogs & $41(18)$ & $27(18)$ & $14(19)$ & \\
\hline Purine analogs + rituximab & $64(28)$ & $42(28)$ & $22(29)$ & \\
\hline Novel agents & $26(11)$ & $16(11)$ & $10(13)$ & \\
\hline Others & $35(15)$ & $24(16)$ & $11(15)$ & \\
\hline Complete response, $\mathrm{n}(\%)$ & $101(56)$ & $67(55)$ & $34(58)$ & NS \\
\hline 10-y overall survival, \% (95\% CI) & $52(47-58)$ & $57(51-64)$ & $42(34-52)$ & 0.003 \\
\hline 10-y risk of Richter syndrome, $\%(95 \% \mathrm{CI})$ & $3(2-5)$ & $3(2-6)$ & $3(1-7)$ & NS \\
\hline \multicolumn{2}{|c|}{10 -y risk of second malignancies, $\%(95 \% \mathrm{CI}) 23(19-28)$} & $23(18-28)$ & $24(17-32)$ & NS \\
\hline
\end{tabular}

$\mathrm{CI}$ : confidence interval; NS: not statistically significant. y: years.

A

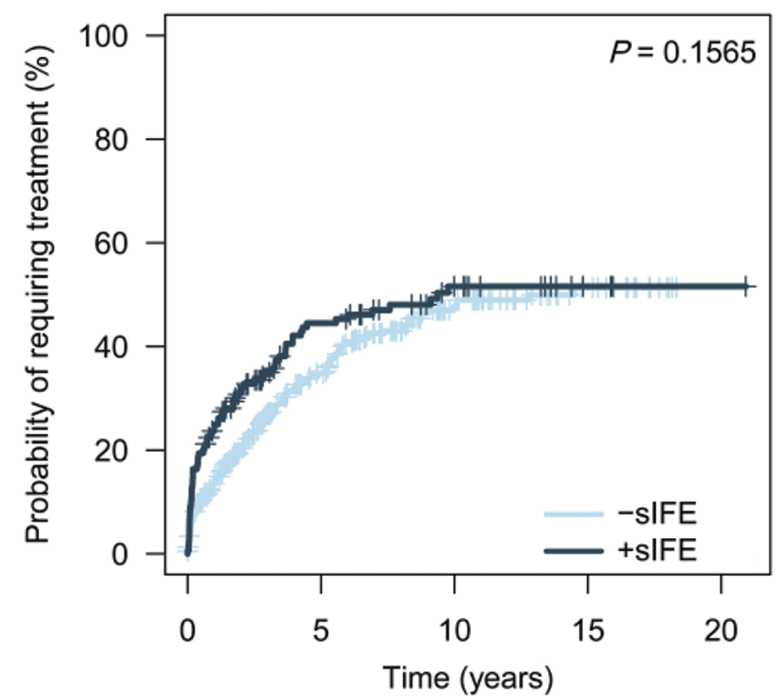

No. at risk

$\begin{array}{rcccc}383 & 153 & 52 & 18 & 0 \\ 165 & 40 & 14 & 3 & 1\end{array}$

B

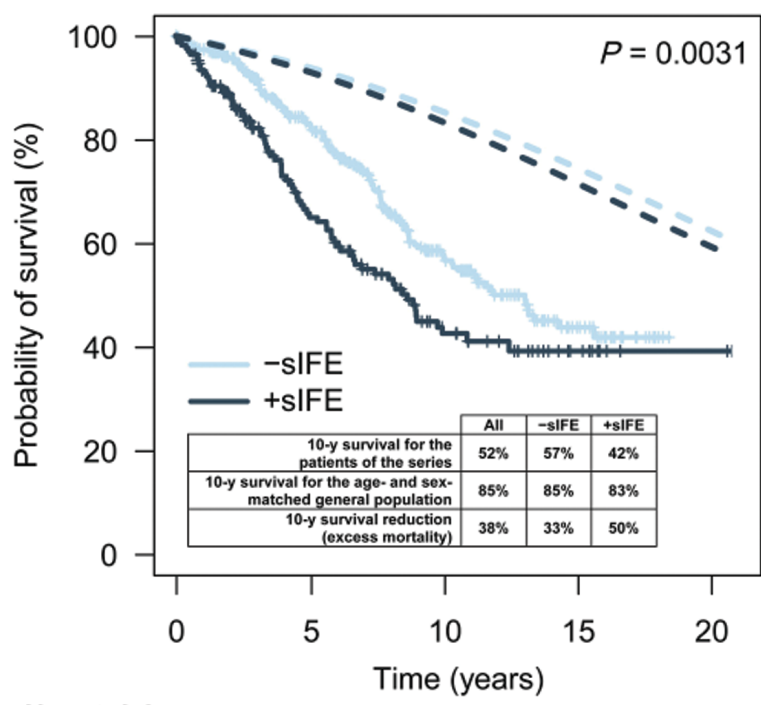

No. at risk

\begin{tabular}{|c|c|c|c|}
\hline 383 & 230 & 88 & 28 \\
\hline 165 & 79 & 35 & 9 \\
\hline
\end{tabular}

Figure 1. Outcomes according to serum immunofixation. (A) Time to first treatment. (B) Overall survival (continuous lines), and survival of the sex- and agematched general population (dashed lines). 
of the 165 patients with a +sIFE can be found in the Online Supplementary Table S1.

Frontline treatment, response, and outcomes are depicted in Table 2. Two-hundred and thirty (42\%) patients received some treatment during follow-up. The median TTFT was 10 years, and no significant differences were seen according to the sIFE (Figure 1A). Frontline regimens were comparable between patients with a negative and positive sIFE, as was the proportion of patients achieving a complete response after treatment.

With a median follow-up of 6.7 years, median OS for the entire cohort was 10.9 years, being significantly shorter for +sIFE compared with -sIFE patients (8.5 vs. 11.9 years; 10 -year OS: 42 vs. $57 \%$; $P=0.003$, Figure 1B). When a two-variable Cox regression, including age (as a quantitative variable) and sIFE, was performed, we observed that both parameters retained independent prognostic impact on OS. Furthermore, despite being significantly older, relative survival analysis showed that excess mortality (survival reduction) with respect to the sex- and age-matched general population was more prominent for patients with a +sIFE ( $50 \%$ at 10 years) compared to that of patients with a - SIFE (33\% at 10 years). In a multivariate model for OS, with 286 cases and 108 events (including age $>60$ years, ECOG PS $\geq 1$, elevated B2M, Rai stage III-IV, unmutated IGHV genes, $\operatorname{del}(17 p)$, CD38 expression $>30 \%$, and a + sIFE), only age, ECOG PS, B2M, and IGHV status retained prognostic impact on OS.

When evaluating OS among patients with a +sIFE according to the heavy chain isotype, no global statistically significant differences were seen. However, when pairwise combinations were performed, biclonal cases had a significantly poorer OS (Online Supplementary Figure $S 1 A$ ), whereas the light chain isotype did not have a significant impact on OS (Online Supplementary Figure S1B). The presence of immunoparesis was evaluated in patients with + sIFE and -sIFE, and it was not an adverse prognostic factor for OS in either of the groups (Online Supplementary Figure S2). No significant differences were found between light chain-concordant and discordant cases with regard to OS. Richter syndrome was seen in 15 patients (3\%). For the entire series, the 10-year RRS was 3\% (95\% Confidence Interval [CI]: 2-5\%), without significant differences according to the sIFE (Online Supplementary Figure S3A). A second tumor was identified in 104 patients $(19 \%)$, and no patient developed multiple myeloma. The 10-year risk of developing a SM was of $23 \%$ for all patients, with similar rates irrespective of sIFE (Online Supplementary Figure S3B).

In conclusion, we found a $30 \%$ prevalence of a + sIFE in our cohort of CLL patients, which is in line with previous studies. ${ }^{5-10}$ Patients with a +sIFE had a more advanced stage and clinical, radiological, biochemical, and genetic poor prognostic markers, as already reported. . $^{5,910}$ Contrary to previous data, ${ }^{9,10}$ however, we observed a clear relationship between a + sIFE and age. In our series, IgM- $\kappa$ constituted the most common isotype, which is in accordance with the report by $\mathrm{Xu}$ and colleagues. ${ }^{9}$ Eleven percent of cases were biclonal, a finding that has been previously interpreted as: i) the malignant transformation occurring at the time of isotype switching from IgM to $\left.\mathrm{IgG},{ }^{12} \mathrm{ii}\right)$ the persistent isotype switching capability of CLL cells, independently of IGHV mutation, ${ }^{13}$ iii) the presence of multiple simultaneous clonal lymphoproliferative disorders, ${ }^{14}$ or iv) the emergence of a subclone secreting a different Ig isotype, due to clonal evolution. The light chain concordance rate was $72 \%$, pointing to the fact that, in most cases, the sMC is a product directly secreted by the tumor population. It had been suggested that concordance was higher in the case of IgM proteins,$^{15}$ and we obtained similar findings. Despite comparable TTFT, frontline regimens, and response, we demonstrated that a +sIFE is a predictor of a poorer OS and relative survival, irrespective of age. However, due to its association with other clinical and biological adverse prognostic features, the sIFE did not retain its negative impact in the multivariate analysis. Biclonal cases had a poorer prognosis, possibly reflecting a higher degree of immunological dysfunction.

The sIFE is positive in one in three CLL patients at diagnosis, and this finding is associated with adverse prognostic baseline features and shorter survival. The study of the sMC in CLL could be an aid to advance in the understanding of B-cell malignancies, anticipate patient outcomes, and eventually tailor therapy.

Pablo Mozas, ${ }^{1 *}$ Juan A. Piñeyroa, ${ }^{1^{*}}$ Ferran Nadeu, ${ }^{2,3}$ Laura Magnano, ${ }^{1,2}$ Andrea Rivero, ${ }^{1}$ Alfredo Rivas-Delgado, ${ }^{1}$ Alex Bataller, ${ }^{1}$ Aleix Fabregat, ${ }^{4}$ Eva Giné, ${ }^{1,2}$ Tycho Baumann, Neus Villamor, 2,3,5 Juan Ignacio Aróstegui, ${ }^{6}$ Marta Aymerich, ${ }^{5}$ Armando López-Guillermo, 1,2,3,7 Elias Campo, 2,3,5,7 and Julio Delgado ${ }^{1,2,3}$

${ }^{1}$ Department of Hematology, Hospital Clinic, Barcelona; ${ }^{2}$ Institut d'Investigacions Biomèdiques August Pi i Sunyer (IDIBAPS),

Barcelona; ${ }^{3}$ Centro de Investigación Biomédica en Red de Cáncer (CIBERONC), Madrid; ${ }^{4}$ Department of Biochemistry and Molecular Biology, Hospital Clinic, Barcelona; ${ }^{5}$ Hematopathology Unit, Department of Pathology, Hospital Clinic, Barcelona; ${ }^{6}$ Department of Immunology, Hospital Clinic, Barcelona and ${ }^{7}$ Universitat de Barcelona, Barcelona, Spain

*PM and JAP contributed equally as co-first authors.

Correspondence:PABLOMOZAS - mozas@clinic.cat

doi:10.3324/haematol.2020.263228

Received: June 17, 2020.

Accepted: October 23, 2020.

Pre-published: November 5, 2020.

Disclosures: no conflicts of interest to disclose.

Contributions: PM and JAP designed the study, collected and analyzed the data, and wrote the manuscript; $F N, L M, A R, A R D$, $A B, A F, E G, T B, N V, J I A, M A, A L G, E C$ and JD contributed to data collection and reviewed the manuscript.

Funding: this study was supported by "Becas de Investigación de la FEHH" (Fundación Española de Hematologia y Hemoterapia) to PM; FN received a pre-doctoral fellowship of the Ministerio de Economia y Competitividad (MINECO, BES-2016-076372); LM received the PI19/00925 grant (Instituto de Salud Carlos III); AL-G and EG received the PI19/00887 grant (Instituto de Salud Carlos III); EC is supported by grants from "La Caixa" Foundation (CLLEvolutionLCF/PR/HR17/52150017), the Instituto de Salud Carlos III, the European Regional Development Fund (FEDER - "Una Manera de Hacer Europa") (PMP15/00007), and is an Academia Researcher of the Institucio Catalana de Recerca i Estudis Avançats (ICREA) of the Generalitat de Catalunya.

\section{References}

1. Azar HA, Hill WT, Osserman EF. Malignant lymphoma and lymphatic leukemia associated with myeloma-type serum proteins. Am J Med. 1957;23(2):239-249.

2. Cox MC, Di Napoli A, Scarpino S, et al. Clinicopathologic characterization of diffuse-large-B-cell lymphoma with an associated serum monoclonal IgM component. PLoS One. 2014;9(4):e93903.

3. Li Y, Wang L, Zhu HY, et al. Prognostic significance of serum immunoglobulin paraprotein in patients with diffuse large B cell 
lymphoma. Br J Haematol. 2018;182(1):131-134.

4. Papageorgiou SG, Thomopoulos TP, Spathis A, et al. Prognostic significance of monoclonal gammopathy in diffuse large B-cell lymphoma. Hematol Oncol. 2019;37(5):634-637.

5. Deegan M, Abraham J, Sawdyk M, Van Slyck E. High incidence of monoclonal proteins in the serum and urine of chronic lymphocytic leukemia patients. Blood. 1984;64(6):1207-1211.

6. Pangalis GA, Moutsopoulos HM, Papadopoulos NM, Costello R, Kokkinou S, Fessas P. Monoclonal and oligoclonal immunoglobulins in the serum of patients with b-chronic lymphocytic leukemia. Acta Haematol. 1988;80(1):23-27.

7. Hansen DA, Robbins BA, Bylund DJ, Piro LD, Saven A, Ellison DJ. Identification of monoclonal immunoglobulins and quantitative immunoglobulin abnormalities in hairy cell leukemia and chronic lymphocytic leukemia. Am J Clin Pathol. 1994;102(5):580-585.

8. Bernstein ZP, Fitzpatrick JE, O'Donnell A, Han T, Foon KA Bhargava A. Clinical significance of monoclonal proteins in chronic lymphocytic leukemia. Leukemia. 1992;6(12):1243-1245.

9. Xu W, Wang YH, Fan L, et al. Prognostic significance of serum immunoglobulin paraprotein in patients with chronic lymphocytic leukemia. Leuk Res. 2011;35(8):1060-1065.

10. El-Hussiny, Ibrahim L, Azmy E, Shamaa S. Prognostic significance of serum monoclonal immunoglobulin in B-chronic lymphocytic leukemia. Egypt J Haematol. 2012;37(4):240.

11. University of California, Berkeley (USA) and MPI for DR (Germany). Human Mortality Database. Available at www.mortality.org

12. Sahota SS, Garand R, Bataille R, Smith AJ, Stevenson FK. V(H) gene analysis of clonally related IgM and IgG from human lymphoplasmacytoid B-cell tumors with chronic lymphocytic leukemia features and high serum monoclonal IgG. Blood. 1998;91(1):238-243.

13. Efremov DG, Ivanovski M, Batista FD, Pozzato G, Burrone OR. IgM-producing chronic lymphocytic leukemia cells undergo immunoglobulin isotype-switching without acquiring somatic mutations. J Clin Invest. 1996;98(2):290-298.

14. Sanchez ML, Almeida J, Gonzalez D, et al. Incidence and clinicobiologic characteristics of leukemic B-cell chronic lymphoproliferative disorders with more than one B-cell clone. Blood. 2003;102(8):29943002.

15. Oian GX, Fu SM, Solanki DL, Rai KR. Circulating monoclonal IgM proteins in B cell chronic lymphocytic leukemia: their identification, characterization and relationship to membrane IgM. J Immunol. 1984;133(6):3396-3400. 